Note

\section{Essential Oil of Scutellaria baicalensis $\mathrm{G}^{\dagger}$}

\section{Katsuya Fukuhara, Takane FujImori, Hitoshi Shigematsu and Akio OHNishi}

Central Research Institute, Japan Tobacco Inc., 6-2, Umegaoka, Midori-ku, Yokohama, Kanagawa 227, Japan

Received November 17, 1986

"Ohgon", a dried preparation of the roots of Scutellaria baicalensis Georgi, is one of the tentative Chinese herbs. It was been used for the treatment of various inflammatory diseases and diarrhoea in Chinese traditional medicine.

Up to now, thirty-eight flavonoids including baicalein and wogonin, 3 sterols and 14 amino acids have been isolated from $S$. baicalensis $\mathrm{G}^{1}{ }^{13)}$ Baicalein and wogonin have been reported to exibit anti-inflammatoric and antiallergic actions. $^{4,5)}$

However, there have been no reports on the essential oil of $S$. baicalensis $\mathrm{G}$. The oil has a good note, and a sweet and aromatic floral odor as well as a medicinal one.

We report here on the components of the essential oil obtained from $S$. baicalensis $\mathrm{G}$.
Crushed and powdered roots, $1200 \mathrm{~g}$ (S. baicalensis $\mathrm{G}$ purchased at a Japanese market), was extracted with ether in a simultaneous distillation extractor (SDE). The ether extract was evaporated to remove the solvent, which afforded the essential oil. A gas chromatogram of the essential oil is presented in Fig. 1. The essential oil $(13.6 \mathrm{~g})$ was chromatographed on a column of silica gel (Merck; silica gel $60,70 \sim 230$ mesh), eluted an $n$-hexane-ether solvent system as an eluate to afford 25 fractions. The respective fractions were eluted with $n$-hexane (fr. $1 \sim 4$, $480 \mathrm{mg}$ ), $2 \%$ ether- $n$-hexane (fr. $5 \sim 8,310 \mathrm{mg}$ ), $5 \%$ ether$n$-hexane (fr. $9 \sim 12,360 \mathrm{mg}$ ), $10 \%$ ether- $n$-hexane (fr. $13 \sim 16,660 \mathrm{mg}$ ), $50 \%$ ether- $n$-hexane (fr. $17 \sim 20,800 \mathrm{mg}$ ), ether (fr. $21 \sim 24,370 \mathrm{mg}$ ) and methanol (fr. 25, $300 \mathrm{mg}$ ). Each fraction was analyzed by GC/MS on a fused silica capillary column (HP 5996A; methyl silicone, $25 \mathrm{~m} \times 0.2 \mathrm{~mm}$ i.d.; $100^{\circ} \mathrm{C} \rightarrow 280^{\circ} \mathrm{C}, 3^{\circ} \mathrm{C} / \mathrm{min}$; ionization vol., $70 \mathrm{eV})$.

Consequently, 81 compounds were identified, as shown in Table I. They were classified into 10 groups as follows: hydrocarbons (21), acids (10), ketones (13), phenols (9), lactones (7), aldehydes (5), alcohols (6), esters (3), ethers (4) and $N$-containing compounds (3). The major constituents of the essential oil were acetopenone (Fig. 1, peak No. 60), (E)-4-phenyl-3-buten-2-one (123), 1-phenyl-1,3butanedione (132), palmitic acid (246) and oleic acid (270).

The essential oil has a medicinal-like odor with a chemical-tone as well as an aromatic floral odor with a sweet-note. Fractions 6 to 16 (ether; $2 \sim 10 \%$ ) had a medicinal-like chemical odor. Fractions 13 to 17 (ether;

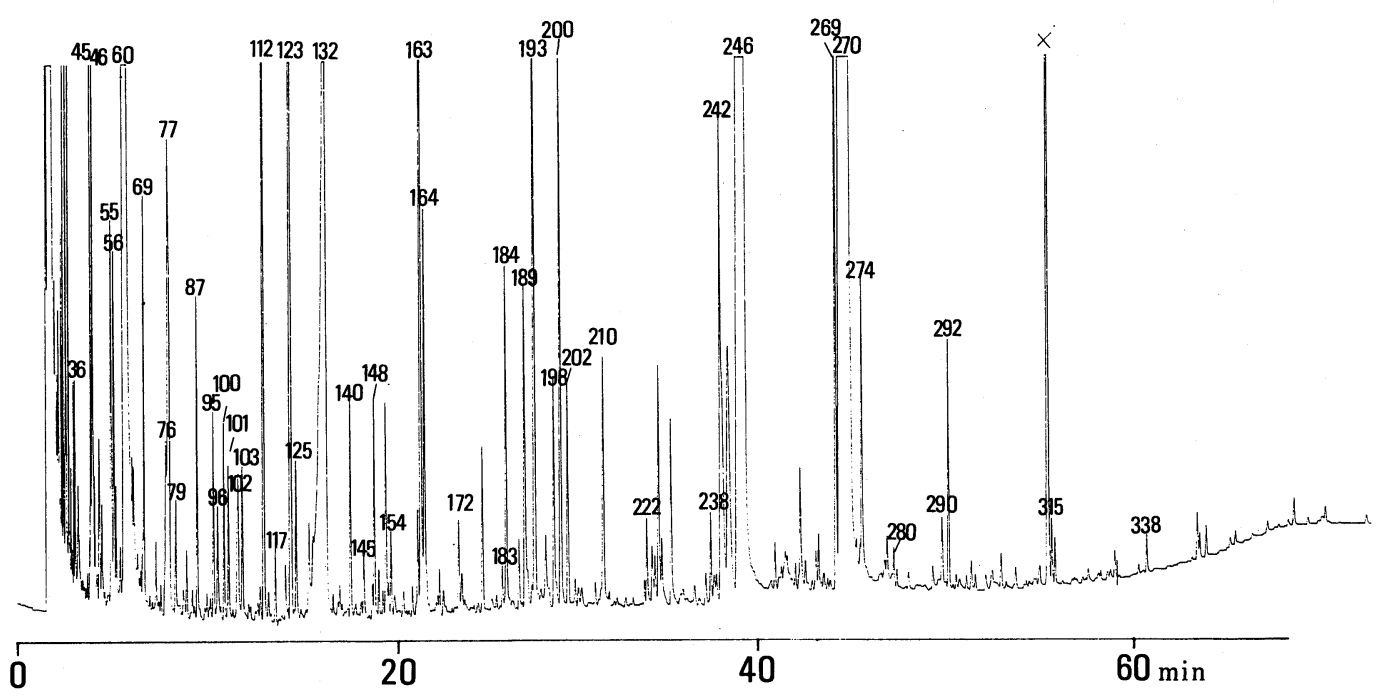

FIG. 1. Gas Chromatogram of the Essential Oil from Scutel-laria baicalensis G.

Conditions: Shimadzu GC-9A; methyl silicone, $25 \mathrm{~m} \times 0.2 \mathrm{~mm}$ i.d.; $100^{\circ} \mathrm{C} \rightarrow 280^{\circ} \mathrm{C}\left(3^{\circ} \mathrm{C} / \mathrm{min}\right) ; \mathrm{He}(1.0 \mathrm{ml} / \mathrm{min})$; FID.

$\dagger^{\dagger}$ This paper was presented at the Annual Meeting of the Agricultural Chemical Society of Japan, Kyoto, April 1986. 
TABLE I. IDENTIFIED COMPOUNDS

\begin{tabular}{|c|c|c|c|c|c|c|c|c|c|}
\hline \multirow{2}{*}{$\begin{array}{c}\text { Peak } \\
\text { No. }\end{array}$} & \multirow{2}{*}{ Compounds } & \multicolumn{3}{|c|}{ Evidence } & \multirow{2}{*}{$\begin{array}{c}\text { Peak } \\
\text { No. }\end{array}$} & \multirow{2}{*}{ Compounds } & \multicolumn{3}{|c|}{ Evidence } \\
\hline & & GC & MS & NMR & & & GC & MS & NMR \\
\hline \multicolumn{5}{|c|}{ Hydrocarbons (21) } & \multicolumn{5}{|c|}{ Esters (3) } \\
\hline 36 & 1,3,5,7-Cyclooctatetraene & & \multicolumn{2}{|l|}{+} & 239 & Methyl palmitate & + & + & \\
\hline 77 & 1,3 -Butadienyl benzene & & \multicolumn{2}{|l|}{+} & 242 & Dibutyl phthalate & + & + & \\
\hline 85 & Dodecane & + & \multicolumn{2}{|l|}{+} & 316 & Dioctyl phthalate & + & + & \\
\hline 117 & Tridecane & + & \multirow{2}{*}{\multicolumn{2}{|c|}{$\begin{array}{l}+ \\
+\end{array}$}} & \multicolumn{5}{|c|}{ Phenols (9) } \\
\hline 134 & $\alpha$-Cubebene & & \multirow{2}{*}{\multicolumn{2}{|c|}{+}} & \multicolumn{2}{|c|}{46 Phenol } & + & + & \\
\hline & $\beta$-Elemene & & & & 57 & $o$-Cresol & + & + & \\
\hline 140 & Tetradecane & + & \multicolumn{2}{|l|}{$\begin{array}{l}+ \\
+\end{array}$} & 61 & $m$ or $p$-Cresol & + & + & \\
\hline 145 & trans-Caryophyllene & & \multirow{2}{*}{\multicolumn{2}{|c|}{+}} & 64 & Guaiacol & + & + & \\
\hline 165 & Pentadecane & + & & & 112 & 4-Ethenyl guaiacol & + & + & + \\
\hline 183 & Hexadecane & $\begin{array}{l}+ \\
+\end{array}$ & \multicolumn{2}{|l|}{$\begin{array}{l}+ \\
+\end{array}$} & 125 & Eugenol & + & + & \\
\hline 104 & Heptadecane & $\begin{array}{l}+ \\
+\end{array}$ & \multicolumn{2}{|l|}{+} & 145 & Isoeugenol & + & + & \\
\hline 211 & $\begin{array}{l}\text { Octadecane } \\
\text { Nonadecane }\end{array}$ & $\begin{array}{l}+ \\
+\end{array}$ & \multirow{2}{*}{\multicolumn{2}{|c|}{$\begin{array}{l}+ \\
+\end{array}$}} & 155 & BHA & & + & \\
\hline $\begin{array}{l}238 \\
250\end{array}$ & $\begin{array}{l}\text { Nonadecane } \\
\text { Eicosane }\end{array}$ & + & & & 163 & BHT & + & + & \\
\hline 269 & Heneicosane & + & + & & Acids & (10) & & & \\
\hline 280 & Docosane & + & + & & 20 & Acetic acid & + & + & \\
\hline 292 & Tricosane & + & + & & 58 & Heptanoic acid & + & + & \\
\hline 304 & Tetracosane & + & + & & 77 & Benzoic acid & + & + & \\
\hline 315 & Pentacosane & + & + & & 79 & Octanoic acid & + & + & \\
\hline 324 & Hexacosane & + & + & & 102 & Nonanoic acid & + & + & \\
\hline 338 & Heptacosane & + & + & & 172 & Decanoic acid & + & + & \\
\hline Keton & les (13) & & & & 210 & Tetradecanoic aicd & + & + & \\
\hline 60 & Acetophenone & + & + & + & 246 & Palmitic acid & + & + & \\
\hline 68 & Maltol & & + & & 270 & Oleic acid & + & + & \\
\hline 77 & 2-Hydroxyacetophenone & & + & & 274 & Stearic acid & + & + & \\
\hline 95 & 4-Phenyl-2-butanone & & + & & Lactor & nes (7) & & & \\
\hline 100 & (Z)-4-Phenyl-3-buten-2-one & & + & + & 54 & 4-Hexanolactone & & + & \\
\hline 121 & 4-Methoxyacetophenone & + & + & & 184 & Butyl phthalide & & + & \\
\hline 132 & (E)-4-Phenyl-3-buten-2-one & + & + & & 189 & Butylidenephthalide & & + & + \\
\hline 132 & $\beta$-Damascone & & + & & 193 & Cnidilide & & + & + \\
\hline 132 & 1-Phenyl-1,3-butanedione & + & + & + & 198 & Senkyunolide & & $\dot{+}$ & \\
\hline 142 & Paeonol & & + & & 200 & Neocnidilide & & + & \\
\hline 147 & Geranyl acetone & + & + & & 202 & Ligustilide & & + & \\
\hline 164 & Ochracin & & + & & Alcoh & ols (6) & & & \\
\hline 222 & Hexahydrofanesylacetone & & + & & 58 & 2-Methyl benzylalcohol & & + & \\
\hline Ethers & (4) & & & & 68 & Linalool & + & + & \\
\hline 49 & 2-Pentyl furan & & + & & 69 & Phenylethylalcohol & + & + & \\
\hline 87 & 2,3-Dihydro benzofuran & & + & & 83 & 4-Terpineol & + & + & \\
\hline 111 & 3-Phenyl furan & & + & & 190 & $\beta$-Eudesmol & & + & \\
\hline 169 & Elemicine & & + & & 290 & Ferruginol & & + & \\
\hline N-Cor & mpounds (3) & & & & Aldeh & ydes (5) & & & \\
\hline 47 & 2-Formyl pyrrole & + & + & & 45 & 5-Methyl-2-furaldehyde & + & + & \\
\hline 57 & Acetyl pyrrole & + & + & & 56 & Phenylacetoaldehyde & + & + & \\
\hline 103 & $(1 H)$-Indole & + & + & & 96 & 4-Methoxybenzaldehyde & + & + & \\
\hline & & & & & 101 & 3-Phenyl-2-propenal & + & + & \\
\hline & & & & & 130 & Vanillin & & + & \\
\hline
\end{tabular}




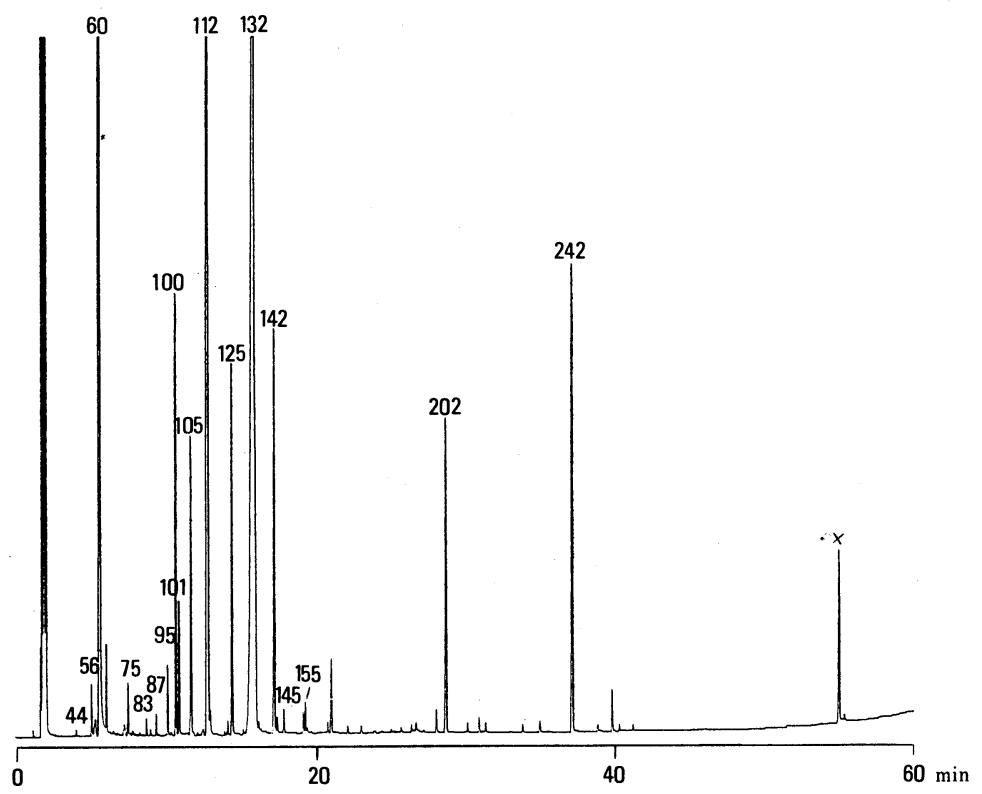

FIG. 2. Gas Chromatogram of Fr. 16.

Compounds: (44) benzaldehyde (tr*); (56) phenylacetoaldehyde (0.6); (60) acetophenone (16.0); (75) 2-methyl benzylalcohol (0.1); (83) 4-terpineol (tr); (87) 2,3-dihydrobenzofuran (0.1); (95) 4-phenyl butanone (0.1); (100) (Z)-4-phenyl-3-butene-2-one (0.9); (101) 3-phenyl propenal (0.3); (103) (1H)-indole (0.8); (112) 4-ethenyl guaiacol (7.1); (125) eugenol (0.9); (132) 1-phenyl-1,3-butanedione (9.1); (142) paeonol (1.3); (145) isoeugenol (0.1); (155) BHA (0.1); (202) ligus-tilide (1.8); (242) dibutyl phthalate (2.6).

$(*)$, ratio of each peak area to the total peak area.

$10 \sim 50 \%$ ) had an aromatic floral odor. Notably, fr. 16 (ether; $10 \%, 130 \mathrm{mg}$ ) had a similar odor to that of the total essential oil. So, the components of fr. 16 were further investigated in order to clarify the key compounds of the odor of $S$. beicalensis $\mathrm{G}$, and 18 compounds were identified (Fig. 2). They comprised 6 ketones, 4 aldehydes, 4 phenols and 4 others. The major constituents of the fraction were acetophenone (Fig. 2, peak No. 60), 4ethenyl guaiacol (112) and 1-phenyl-1,3-butanedione (132), the odors of the respective major compounds being hawthron-like, herbaceous and floral. However, no key compound that had the same odor as the essential oil itself was found contribute to the characteristic odor of the essential oil.

\section{REFERENCES}

1) T. Tomimori, H. Jin, Y. Miyaichi, S. Toyofuku and T. Namba, Yakugaku Zasshi, 105, 148 (1985).

2) M. Takido, M. Aimi, S. Takahashi, S. Yamanouchi, H. Torii and M. Dohi, Yakugaku Zasshi, 95, 108 (1975).

3) S. Takagi, M. Yamaki and K. Inoue, Yakugaku Zasshi, 100, 1221 (1980).

4) M. Kubo, H. Matsuda, M. Tanaka, Y. Kimura, H. Okuda, M. Higashino, T. Tani and K. Namba, Chem. Pharm. Bull., 32, 2724 (1984).

5) A. Koda, H. Nagai and H. Wada, Folia Pharmacol. Japan, 66, 471 (1970). 